\title{
A Peculiar Type II QSO Identified via Broad-band Detection of Extreme Nebular Line Emission $\star \dagger$
}

\author{
Yu-Heng Lin ${ }^{1,2} \neq$, Claudia Scarlata ${ }^{2}$, Matthew Hayes ${ }^{3}$, Anna Feltre $^{4}$, Stephane Charlot ${ }^{5}$, Angela Bongiorno ${ }^{6}$, \\ Petri Väisänen ${ }^{7,8}$, and Moses Mogotsi ${ }^{7,8}$ \\ ${ }^{1}$ School of Physics and Astronomy, University of Minnesota, 116 Church St SE, Minneapolis, MN 55455, USA \\ ${ }^{2}$ Minnesota Institute for Astrophysics, University of Minnesota, 116 Church St SE, Minneapolis, MN 55455, USA \\ ${ }^{3}$ Stockholm University, Department of Astronomy and Oskar Klein Centre for Cosmoparticle Physics, AlbaNova University Centre, SE-10691, Stockholm, Sweden \\ ${ }^{4}$ INAF - Osservatorio di Astrofisica e Scienza dello Spazio di Bologna, Via P. Gobetti 93/3, 40129 Bologna, Italy \\ ${ }^{5}$ Sorbonne Université, CNRS, UMR7095, Institut d'Astrophysique de Paris, F-75014, Paris, France \\ ${ }^{6}$ INAF - Osservatorio Astronomico di Rioma, Via Frascati 33 00074, Monteporzio Catone, Italy \\ ${ }^{7}$ South African Astronomical Observatory, P.O. Box 9, Observatory, 7935, Cape Town, South Africa \\ ${ }^{8}$ Southern African Large Telescope, P.O. Box 9, Observatory, 7935, Cape Town, South Africa
}

Accepted 2021 October 11. Received 2021 September 27; in original form 2021 May 5

\begin{abstract}
We present S82-20, an unusual redshift $\approx 3$ object identified in SDSS-Stripe 82 broad-band images. The rest-frame ultraviolet spectrum of S82-20 shows emission lines from highly ionized species, including He II $\lambda 1640$, and the $\mathrm{C}_{\text {IV }} \lambda \lambda 1548,1550$ and O vi $\lambda \lambda 1032,1038$ doublets. The high Ly $\alpha$ luminosity $\left(3.5 \times 10^{44} \mathrm{erg} \mathrm{s}^{-1}\right)$, the high emission line equivalent widths $(>200 \AA ̊$ for $\operatorname{Ly} \alpha)$, the FWHM of the emission lines $\left(<800 \mathrm{~km} \mathrm{~s}^{-1}\right)$, and the high ionization $\mathrm{O}$ vi line strongly support the interpretation that S82-20 is a Type II QSO. However, photoionization models using Type II QSO do not fully explain the measured C IV/He II line ratio, which requires either some contribution from star-formation or high velocity shocks. Additionally, S82-20 is not detected at wavelengths longer than $2 \mu \mathrm{m}$, in tension with the expectation of isotropically IR emission of a luminous QSO. We consider the possibility that S82-20 is a rare example of a changing-look QSO, observed in a temporarily low state, where the broad line region has faded, while the narrow line region still emits emission line. Otherwise, it may be a rare case of the short phase of the life of a massive galaxy, in which active star formation and accretion onto a supermassive black hole coexist.
\end{abstract}

Key words: galaxies:active - quasars: emission lines - galaxies: high-redshift

\section{INTRODUCTION}

Observations suggest that the cosmic evolution of the star formation rate and black hole accretion rates are similar: both increase with redshift, reaching a peak at redshift $z \simeq 2$, and declining in the earlier universe (e.g., Silverman et al. 2008; Madau \& Dickinson 2014; Vito et al. 2017). This similarity, together with the fact that supermassive black holes (SMBHs) are ubiquitous in the centers of galaxies in the local universe as well as the well known scaling relations between galaxies' properties and black hole mass (Magorrian et al. 1998) suggest the existence of a tight connection between the growth of SMBHs and the evolution of their host galaxy (Ferrarese \& Merritt 2000; Gebhardt et al. 2000; Madau \& Dickinson 2014). It has been suggested that this connection results from the fact that the $\mathrm{BH}$ accreting phase contributes to the regulation of galaxy growth, possibly via feedback. On one hand, $\mathrm{BH}$ activity traces gas accretion; on the other hand, this phase is also a source of feedback (for example, through powerful winds) and may be able to quench star-formation in massive haloes (Hopkins et al. 2008; Richardson et al. 2016).

SMBHs can be identified and studied while they are accreting

† E-mail: lin00025@umn.edu material. This phase is called the Active Galactic Nucleus (AGN) phase. In the unification model, the accretion disk is surrounded by an obscuring torus (Antonucci 1993; Urry \& Padovani 1995). This simple model explains a vast range of observations, as the result of viewing conditions. In this context, AGN are divided into Type I and Type II AGN, depending on whether or not the broad line region (which is generated in the centermost regions) is visible. For Type II, obscured AGN, the line of sight to the broad-line region is blocked by the dusty torus, while the narrow-line region, which can extend up to scales of several kpcs, can still be observed.

In addition to the unification model, the obscured phase can be explained with the merger-driven scenario (Di Matteo et al. 2005; Hopkins et al. 2006; Hopkins et al. 2008). In this scenario, gasrich galaxy mergers drive inflows, producing central starbursts and fueling the growth of the SMBH. Type I and Type II AGN are then objects observed in different evolutionary phases rather than from different angles . During the obscured phase, the nucleus is buried in the surrounding inflowing materials. After the energy released in the accretion process dominates in the central regions, radiative feedback can blowout the surroundings and the active galaxy evolves into an unobscured quasar. Some AGNs were observed to dramatically change their broad emission component, with the spectra varying 
between Type I, Type II, or the intermediate Type 1.5, 1.8 (Penston \& Pérez 1984; Veilleux \& Osterbrock 1987; Elitzur et al. 2014). The variation of these so called "changing-look" AGN can be driven by different mechanisms including: (1) variable obscuration due to a moving patchy dust torus (Elitzur 2012); (2) variable accretion rate in the central engine (Elitzur et al. 2014; LaMassa et al. 2015); (3) tidal disruption events (e.g., Eracleous et al. 1995).

High redshift Type I quasars are routinely identified in optical surveys thanks to their colors (Padovani et al. 2017). Spectroscopic confirmation is then easily achieved, given their brightness, and easyto-detect broad emission lines in the rest-frame UV. Optical surveys, however, are less successful at identifying Type II QSOs, since the AGN continuum, as well as the broad emission lines, are completely obscured along our line of sight. Therefore, the identification of Type II QSOs is a multi-step process that requires spectroscopic follow-up to determine the nature of the source responsible for the excitation of the gas.

Many surveys have searched for Type II QSOs, using criteria based on the interaction between photons and the obscuring material. Typically, Type II obscured accretion is identified in the following ways: $\mathrm{X}$-ray observations detect the obscured high-energy photons, mid- to far-IR observations directly probe the thermal re-emission of dust, and optical spectroscopic observations search for narrow emission lines with no broad components (e.g., Lacy et al. 2004; Donley et al. 2012; Stern et al. 2012; Assef et al. 2013; Hainline et al. 2014; Alexandroff et al. 2013; Hickox et al. 2017). Recently, however, it has become clear that obscured accretion may look quite different, depending not only on the column density of obscuring material, but also on the intrinsic luminosity (e.g., Ueda et al. 2003; Hasinger 2004) and the BH accretion rate (e.g., Fabian 1999). Additionally, different mechanisms may originate obscured activity (Rigby et al. 2006; Li et al. 2020). Thus, alternative methods to identify obscured quasars are important to truly constrain the variety of the AGNs and their correlations to the host galaxies.

Spectroscopic searches for high redshift quasars use the presence of bright and narrow emission lines in the rest-frame UV to identify Type II quasar candidates (e.g., Alexandroff et al. 2013, who applied a cut at line widths Full Width Half Maximum of $2000 \mathrm{~km} \mathrm{~s}^{-1}$ ). These samples result in moderately obscured quasars, as revealed by follow-up rest-frame optical spectroscopy (Greene et al. 2014; Hickox \& Alexander 2018; Burtscher, L. et al. 2016; Schnorr-Müller et al. 2016).

A promising complementary technique to identify Type II QSOs is to search for objects with bright UV emission lines but a faint continuum. This is because, in the unification model, the bright continuum from the accretion disk and the broad-line region would be fully obscured by the torus along the line of sight, while the kpcscale narrow-line region would still shine unaffected (e.g., Antonucci 1993; Urry \& Padovani 1995; Nakajima, K. et al. 2018). Narrow, high EW emission lines can however also be a feature of young metal-poor star-forming galaxies (SFGs). High EWs of the Ly $\alpha$ emission line (e.g., larger than few hundreds $\AA$ ) are also expected for metal-free Population III stars (Schaerer, D. 2003; Raiter et al. 2010).

In a search for extreme emission line galaxies, we have identified a promising Type II QSO candidate. We are conducting a broadband search for emission line galaxies, that covers $200 \square^{\circ}$ (Mehta et al. in prep) within the SDSS Stripe 82 (Annis et al. 2014; Jiang et al. 2014). Candidates are selected if they show a $g$-band flux excess, that we assume is a result of a strong emission line. As part of the spectroscopic followup campaign, we have discovered a highly unusual object, hereafter S82-20. This galaxy, at $z=3.082$, is characterized by a narrow emission line spectrum $(\mathrm{FWHM} \approx 700 \mathrm{~km}$ $\mathrm{s}^{-1}$ ), showing a strong Ly $\alpha$ emission, and additional lines from the C IV $\lambda \lambda$ 1548, $1550 \AA$ A doublet, He II $\lambda 1640 \AA, C_{\text {III] }} \lambda \lambda 1907,1909 \AA$ doublet, and, most remarkably, the O vi $\lambda \lambda$ 1032, $1038 \AA$ doublet. The observed line ratios cannot distinguish between AGN and SF dominated excitation, although photoionization by stars is highly unlikely given the presence of the $\mathrm{O}$ vi lines that need $\approx 10$ Rydbergs to excite. Additionally, the galaxy is not detected at long wavelengths ( $\lambda>2 \mu \mathrm{m}$ rest-frame), arguing against the obscured Type II QSO origin.

This paper is organized as follows. The data and data reduction, the measurements, and the analysis of the photometric and spectroscopic data are presented in Section 2. In Section 3, we present the calculation of the emission line ratios from photoionization and shocks models. Section 4 presents the results which are discussed in Section 5. The conclusions of this work are presented in Section 6. Thorough out the paper, we assume the Lambda cold dark matter $(\Lambda \mathrm{CDM})$ cosmology with the following parameters: $H_{0}=70 \mathrm{~km} \mathrm{~s}^{-1}$ $\mathrm{Mpc}^{-1} ; \Omega_{m}=0.3 ; \Omega_{\Lambda}=0.7$.

\section{OBSERVATIONS AND DATA ANALYSIS}

In this section, we describe the data available on S82-20. Section 2.1 presents the spectroscopic data and the data reduction. Section 2.2 presents the measurements of the emission line fluxes, equivalent width, and velocity width. Section 2.3 describes available archival imaging in the optical through far-IR as well as the measurements of S82-20 total fluxes (or upper limit) at various wavelengths.

\subsection{Spectroscopic Observations}

S82-20 [RA=23:48:11.86, DEC=-00:11:47.85 (357.04941596, $0.19661512)]$ was observed in three occasions. A first confirmation spectrum in 2013 with the 200-inch Hale Telescope, a second higher resolution spectrum with the $11 \mathrm{~m}$ Southern African Telescope (SALT, Buckley et al. 2006) in 2018, and finally a full spectrum extending the rest-frame coverage to the $\mathrm{C}_{\mathrm{III}}$ ] lines, using the Large Binocular Telescope (MODS/LBT), in 2020. The following sections describe the data and data reduction.

\subsubsection{Palomar observations}

A first optical spectrum was acquired with the Double-Beam Spectrograph (DBSP, Oke \& Gunn 1982) mounted on the Hale $5 \mathrm{~m}$ telescope at Palomar Observatory in October 2013. We used the D55 dichroic to split the light at $\sim 5500 \AA$ into a blue and a red channel. The red spectrum, acquired with the $600 / 10000$ lines $\mathrm{mm}^{-1}$ grating, covers the wavelength range between $6000 \AA$ and $8500 \AA$. The blue spectrum was acquired with the $600 / 4000$ lines $\mathrm{mm}^{-1}$ grating, and covers the wavelength range between $3500 \AA$ and $5500 \AA$. We used a 1 '. 5 wide slit, resulting in a spectral resolution of $8 \AA$ (i.e., $\sim 500 \mathrm{~km} \mathrm{~s}^{-1}$ ) at $5000 \AA$. We obtained two exposures of 1,800 s each, with seeing full width half maximum (FWHM) of $\sim 1$.' $^{\prime} 0$.

We performed the basic steps of the data reduction (bias subtraction, flat-field normalization, wavelength calibration, and spectral extraction) using standard IRAF packages (Tody 1986). The observing night was clear, but not photometric. Therefore, we removed the wavelength-dependent instrumental response using the sensitivity curve derived from the spectrum of the standard star BD+17_4708 observed during a photometric night in the same observing run. The absolute flux calibration was then performed normalizing the 
red and blue spectra to the total SDSS $r$ - and $g$-band magnitudes, respectively.

The final extracted Hale spectrum is shown in Figure 1.

\subsubsection{SALT observations}

S82-20 was observed in service mode using the Robert Stobie Spectrograph (RSS, Burgh et al. (2003)) at the SALT telescope. Observations were acquired between June and September, 2018, over 6 nights. Data from each night included a 1064 second exposure in long slit mode, with a slit width of $1 .{ }^{\prime \prime} 5$. The slits were oriented to cover both the target object S82-20, as well as an alignment star, which was later used to flux calibrate the spectrum. The detector of the RSS spectrograph (Burgh et al. 2003) consists of a mosaic of three CCDs, with pixel size of $15 \mu \mathrm{m}$, corresponding to a spatial resolution of 0 .' 26 per pixel using the $2 \times 2$ binning. The seeing FWHM was 1 .' 5 during the observations. We used the RSS PG2300 grating with a tilt angle 32.375 degrees which provides the wavelength coverage from $4100 \AA$ to $5140 \AA$, with a spectral resolution of 2500 .

The basic data reduction steps of overscan, bias and gain correction, crosstalk, mosaicking of the CCDs, and merging of the amplifiers were performed using the SALT primary data reduction pipeline (Crawford et al. 2010) ${ }^{1}$. Further analysis, including flat-fielding, illumination, wavelength calibration, background sky subtraction, removal of cosmic rays, atmospheric extinction correction, and flux calibration, was performed using the IRAF package. The background was subtracted by fitting a second-order polynomial to each column along the cross-dispersion direction. We removed the cosmic ray hits using the L.A.Cosmic ${ }^{2}$ spectroscopic version on IRAF. After correcting each exposure for atmospheric extinction, we combined them into a single image to increase the signal to noise ratio.

We extract S82-20 and the reference star separately, using apertures 3 .' 0 and 6.' 3 wide, respectively. To perform the flux calibration of the SALT spectrum we used the reference star observed in the same slit. This star was observed spectroscopically as part of the SDSS survey in February 2008, and the spectrum is available from Data Release 14. We derived the wavelength dependent instrument sensitivity function by comparing the RRS and SDSS spectra of the reference star. We checked that the reference star is not variable using data from PanSTARRS (Chambers et al. 2019).

The final extracted Hale spectrum is shown in Figure 2.

\subsubsection{LBT observation}

We observed S82-20 with the Multi-Object Double Spectrographs (MODS, Pogge et al. 2010) mounted on the Large Binocular Telescope (LBT) on October 12th, 2020 for a total observing time of 1 hour. The one-hour integration time was split into three exposures of 1200 seconds each. The data were taken using MODS1 and MODS2 in long-slit dual beam G400L/G670L grating mode, using a 1.'0 wide slit. The resulting spectrum covers the wavelength range from $3200 \AA$ to $10,000 \AA$, with a resolving power of $\mathrm{R}=2000$. The observing conditions were clear and the seeing FWHM was $\sim 0 .{ }^{\prime \prime} 9$ during the observations.

We performed the primary detector calibration including bias, flat field and fixing of the bad columns using the public python program

\footnotetext{
1 http://pysalt.salt.ac.za/

2 http://www.astro.yale.edu/dokkum/lacosmic/
}

Table 1. S82-20 emission line properties

\begin{tabular}{|c|c|c|c|}
\hline & SALT & Palomar & LBT \\
\hline Line & $\begin{array}{l}\text { Luminosity } \\
10^{43} \mathrm{erg} / \mathrm{s}\end{array}$ & $\begin{array}{l}\text { Luminosity } \\
10^{43} \mathrm{erg} / \mathrm{s}\end{array}$ & $\begin{array}{c}\text { Luminosity } \\
10^{43} \mathrm{erg} / \mathrm{s}\end{array}$ \\
\hline $\operatorname{Ly} \alpha$ & $35.45 \pm 0.19$ & $39.45 \pm 1.12$ & $36.10 \pm 0.21$ \\
\hline $\mathrm{OVVI}$ & $3.95 \pm 0.28$ & $3.7 \pm 0.1$ & $3.70 \pm 0.17$ \\
\hline $\mathrm{Nv}$ & $1.23 \pm 0.13$ & $<1$ & $1.34 \pm 0.12$ \\
\hline Civ & - & $10.0 \pm 1.0$ & $8.67 \pm 0.05$ \\
\hline He II & - & $1.6 \pm 0.2$ & $1.52 \pm 0.04$ \\
\hline O III] & - & - & $0.38 \pm 0.04$ \\
\hline $\left.\mathrm{C}_{\mathrm{III}}\right]$ & - & - & $2.04 \pm 0.07$ \\
\hline Line & $\begin{array}{l}\text { FWHM } \\
\mathrm{km} \mathrm{s}^{-1}\end{array}$ & $\begin{array}{l}\text { FWHM } \\
\mathrm{km} \mathrm{s}^{-1}\end{array}$ & $\begin{array}{l}\text { FWHM } \\
\mathrm{km} \mathrm{s}^{-1}\end{array}$ \\
\hline $\operatorname{Ly} \alpha$ & $734.8_{-12.8}^{+8.5}$ & $741_{-10}^{+21}$ & $774.2_{-8.5}^{+4.1}$ \\
\hline OvI & $662.0 \pm 42.8$ & $726 \pm 88$ & $635.8 \pm 17.9$ \\
\hline Civ & - & $819 \pm 85$ & $838.0 \pm 10.7$ \\
\hline He II & - & - & $575.9 \pm 14.6$ \\
\hline $\left.\mathrm{C}_{\mathrm{III}}\right]$ & - & - & $775.9_{-122.3}^{+174.7}$ \\
\hline Line & $\begin{array}{c}\mathrm{EW}^{a} \\
\AA\end{array}$ & $\begin{array}{c}\mathrm{EW}^{a} \\
\AA\end{array}$ & $\begin{array}{c}\mathrm{EW}^{a} \\
\AA\end{array}$ \\
\hline $\operatorname{Ly} \alpha$ & - & - & $754.3 \pm 165.2$ \\
\hline $\operatorname{Ly} \alpha^{b}$ & $275.15 \pm 21.8$ & $306.2 \pm 27.5$ & $280.3 \pm 22.2$ \\
\hline $\mathrm{O}_{\mathrm{vI}}^{b}$ & $30.63 \pm 3.2$ & $28.7 \pm 2.2$ & $28.7 \pm 2.6$ \\
\hline $\mathrm{N} \mathrm{v}^{b}$ & $9.5 \pm 1.3$ & - & $10.4 \pm 1.2$ \\
\hline $\mathrm{C}_{\text {IV }}$ & - & $229.6 \pm 44.8^{c}$ & $192.4 \pm 16.7$ \\
\hline He II & - & $36.7 \pm 7.4^{c}$ & $29.7 \pm 2.5$ \\
\hline $\left.\mathrm{C}_{\mathrm{III}}\right]$ & - & - & $38.1 \pm 4.2$ \\
\hline
\end{tabular}

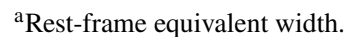

${ }^{\mathrm{b}}$ Continuum derived from $g$ band imaging.

${ }^{\mathrm{c}}$ Continuum derived from $r$ band imaging.

modsCCDRed (Pogge 2019). The following steps (wavelength calibration, sky subtraction, spectral extraction, and flux calibration) were performed with the modsIDL pipeline (Croxall \& Pogge 2019). The spectrum was extracted using a 2 '. $^{\prime}$ aperture. To derive the wavelength dependent instrument response we used the spectrum of the standard star BD+28 4211 observed during the same night. The absolute flux calibration was then performed normalizing the red and blue spectra to the total SDSS S82-20 $r$ - and $g$-band magnitudes, respectively. We combined the MODS1 and MODS2 spectra via a weighted sum, with the weights equal to the variance of the continuum around the Ly $\alpha$ emission for the blue channel and around C IV emission for the red channel.

The final extracted MODS/LBT spectrum is shown in Figure 3.

\subsection{Emission line measurements}

The first spectrum obtained at the Hale telescope confirms that the color excess in the $g$-band is due to a strong Ly $\alpha$ emission line, and that the object is at $z=3.082$. In addition to the detection of strong Ly $\alpha$, the Hale spectrum reveals the presence of high ionization lines, including the $\mathrm{C}_{\mathrm{IV}} \lambda \lambda 1549,1551 \AA$ (hereafter $\mathrm{C}_{\text {IV }}$ doublet) and O vi $\lambda \lambda 1032,1038 \AA$ (hereafter O vi doublet). The spectrum additionally indicates the presence of $\mathrm{He}$ II and $\mathrm{N} v$ emission lines, although the signal-to-noise ratio in these faint lines is low. The SALT spectrum confirms the detection of the $\mathrm{O}$ vi doublet, but does not extend red enough to cover the $\mathrm{C}$ IV doublet and $\mathrm{He}_{\mathrm{II}}$ lines.

In addition to the bright $\mathrm{O}_{\mathrm{VI}}, \mathrm{Ly} \alpha$, and $\mathrm{C}_{\mathrm{IV}}$ lines, the LBT 


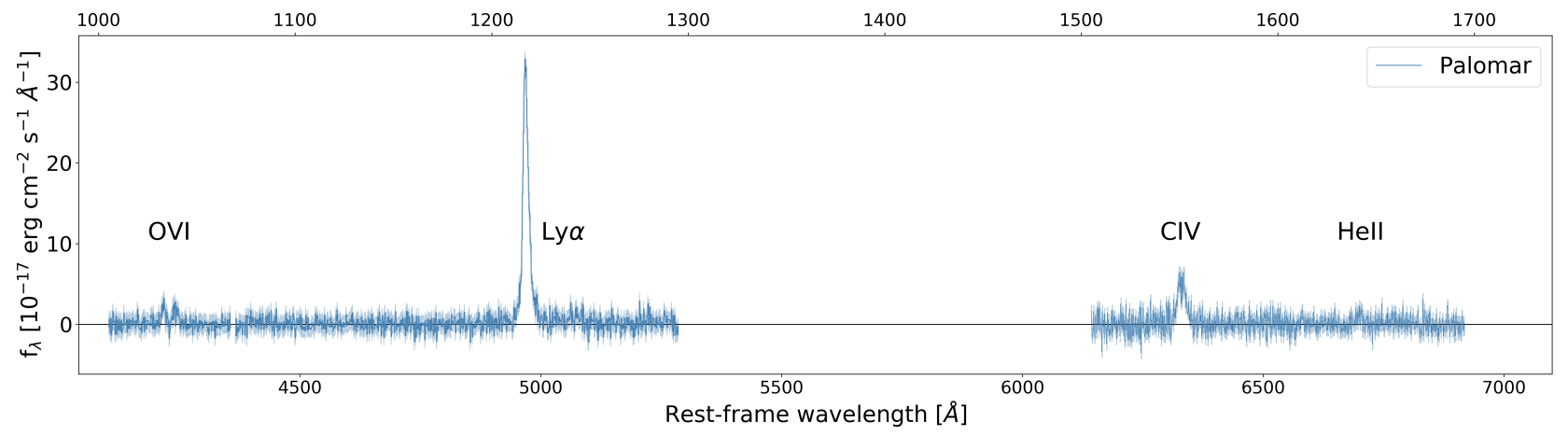

Figure 1. Spectrum of S82-20 obtained with the Hale 5m telescope. In addition to Ly $\alpha$, the spectrum shows a clear detection of the C IV and the O vi doublets.

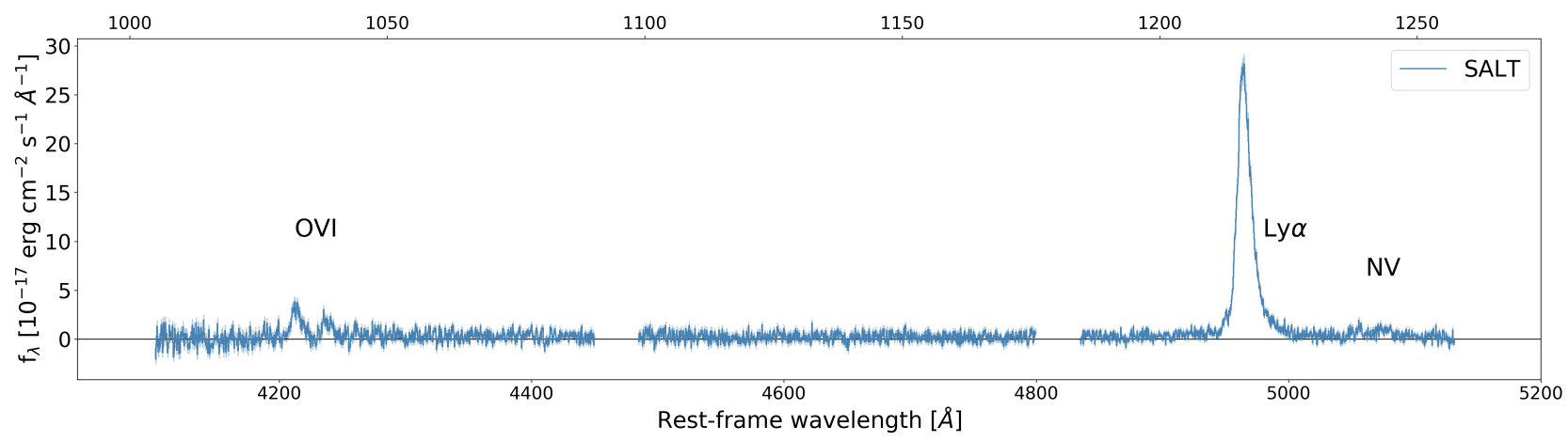

Figure 2. Spectrum of S82-20 obtained with SALT telescope. Note that the different setup provides higher resolution but a shorter wavelength range than in Figure 1

spectrum, shown in Figure 3, clearly reveals the presence of He ${ }_{I I} \lambda 1640$ (hereafter $\mathrm{He}_{\mathrm{II}}$ ), $\left.\mathrm{O}_{\mathrm{III}}\right] \lambda 1666$ (hereafter $\left.\mathrm{O}_{\mathrm{III}}\right]$ ), and the $\left.\mathrm{C}_{\text {III }}\right] \lambda \lambda 1907,1909$ doublet (here after $\mathrm{C}_{\text {III }}$ ). We measured the flux of the emission lines by directly integrating the spectral flux density over the wavelength range of the lines shown in Figure 3, after subtracting the continuum. We derived the continuum for each line by averaging the flux density over the spectral regions indicated in Figure 3. For most lines we use two $25 \AA$-wide regions, one on each side of the emission line. For $\operatorname{Ly} \alpha$, in order to minimize biases introduced by the Ly $\alpha$ forest, we only measure the continuum on the red side of the line. Even though we find that the continuum is not detected at more than $3 \sigma$, we properly account for its contribution in the line flux error calculation.

For the emission lines in which the continuum was not detected in the spectrum (see Table 1), we compute the observed equivalent width (EW) using the continuum derived from the emission-linecorrected broad band fluxes as follows. Assuming that the $g$ and $r$ bands are dominated by the $\mathrm{Ly} \alpha+\mathrm{O}_{\mathrm{VI}}$ and $\mathrm{C}_{\mathrm{IV}}$ emission lines, respectively, we can write the continuum flux density $\left(f_{\lambda}^{\text {cont }}\right)$ as:

$f_{\lambda}^{\text {cont }}=f_{g, r}-\frac{F_{\text {line }}}{W_{g, r}}$,

where $f_{g, r}$ are the flux density computed from the $g$ and $r$ band magnitudes, $W_{g, r}$ are the FWHM of the $g$ and $r$ band filters, and $F_{\text {line }}$ is the total flux of the emission line. We measure continuum levels of $f^{\text {cont }}(g)=3.6 \pm 0.3 \times 10^{-18} \mathrm{erg} \mathrm{cm}^{-2} \mathrm{~s}^{-1} \AA^{-1}$ and $f^{\text {cont }}(r)=$ $1.2 \pm 0.2 \times 10^{-18} \mathrm{erg} \mathrm{cm}^{-2} \mathrm{~s}^{-1} \AA^{-1}$. These values are consistent with the upper limits derived from the spectra.

Finally, to compute the FWHM of the emission lines we proceed in two different ways for Ly $\alpha$ and the remaining, weaker lines . For Ly $\alpha$ we compute the FWHM numerically, by finding the width of the profile where the value is half of the maximum value. The error on the FWHM is then computed via a Monte Carlo simulation. We generate 1000 realizations of the $\operatorname{Ly} \alpha$ profile by randomizing the spectrum within $\pm \sigma_{\text {cont }}$ at each wavelength. For each realization, we compute the line FWHM, and then estimate the error as the standard deviation of the distribution of the 1000 realizations. For the lower S/N emission lines, we measure the FWHM by fitting a Gaussian profile to the observed spectra. The $\mathrm{C}$ IV and $\mathrm{O}$ vi doublets have the intrinsic 2:1 line ratio in the low electron density and optical thin environment $\left(n_{e} \tau_{0}<10^{16} \mathrm{~cm}^{-3}\right.$, Hamann et al. 1995; Kramida et al. 2019). Since we measure the $\mathrm{O}$ vi doublet line ratio $\simeq 2: 1$, we fix the $\mathrm{C}$ IV doublet line ratio as $2: 1$ and fit with a double Gaussian profile. The flux ratio of $\left.\left.\mathrm{C}_{\text {III }}\right] 1907 / \mathrm{C}_{\text {III }}\right] 1909$ varies from 1.53 to 0 with electron density (Keenan et al. 1992). While the line profile in our spectrum is not clear to distinct the doublet. We generate 10,000 realizations of the $\mathrm{C}_{\mathrm{III}}$ ] profile by randomizing the spectrum within $\pm \sigma_{\text {cont }}$ at each wavelength. The FWHM of $\mathrm{C} \mathrm{III]} \mathrm{by} \mathrm{fitting} \mathrm{a} \mathrm{single}$ Gaussian profile is $775.9_{-122.3}^{+174.7} \mathrm{~km} \mathrm{~s}^{-1}$.

Table 1 presents the measurements performed on the available spectra. For the doublets ( $\mathrm{O}_{\text {VI }} \mathrm{C}_{\mathrm{IV}}$ and $\mathrm{C}_{\mathrm{III}}$ ) we report the sum of the two lines in the doublet. In the following analysis, we use the emission line fluxes measured on the LBT spectra, unless we state otherwise. 

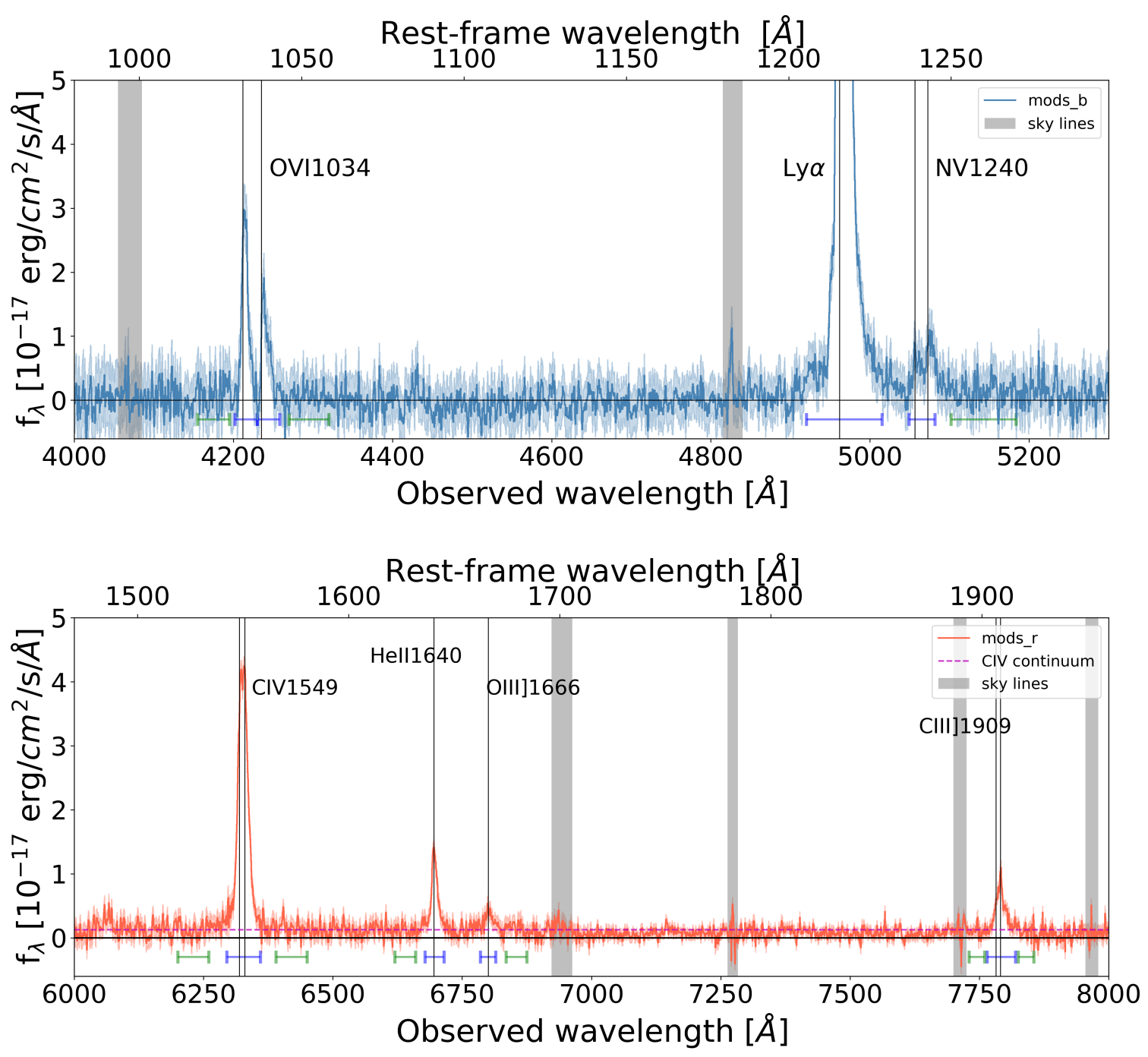

Figure 3. Spectrum from MODS/LBT. The main emission lines identified are marked. The blue bars are the integration range for emission line flux, and the green bars are the wavelength range for emission line continuum level.

\subsection{Photometric measurements}

The field of S82-20 was imaged at optical to mid-infrared wavelengths with a number of instruments. The measurements of the total flux in the different bands, described below, are presented in Table 2. We show the resulting spectral energy distribution in Figure 4.

At the optical wavelengths, the field was observed as part of the SDSS Stripe 82 campaign (Annis et al. 2014). The fluxes in the $u, g, r, i$ and $z-$ bands were measured using circular apertures of 3.' 56 radius. In Figure 4 we show both the direct measurements (filled circles), as well as the values corrected for the contribution of the emission lines (open circles). The $g-$ and $r$-band are clearly affected by the presence of $\mathrm{Ly} \alpha$ and $\mathrm{C}_{\mathrm{IV}}$, respectively. At longer wavelengths, the field of S82-20 is covered by the Spitzer-IRAC Equatorial Survey (SpIES) program $3.6 \mu \mathrm{m}$ and $4.5 \mu \mathrm{m}$ (Timlin et al. 2016), by the Wide-field Infrared Survey Explorer at 12, and $22 \mu \mathrm{m}$
(Wright et al. 2010), and by the Herschel telescope at 250, 350, and $500 \mu \mathrm{m}$ as part of the HerMES Large Mode Survey (HeLMS) Survey (Asboth et al. 2016).

The co-added Spitzer images were downloaded from the IRSA archive and the fluxes were calculated within circular apertures. The object is clearly detected in both Spitzer bands as shown in the top panels of Figure 4. To compute the total flux in the IRAC bands we used circular apertures with a 2 '. 4 radius. The background was evaluated within a circular annulus with inner radius of 7. .' 2 and outer radius of $12^{\prime \prime}$ and subtracted from the total flux measured within the aperture.

We used the WISE/NEOWISE Coadder ${ }^{3}$ tool to create a deeper co-add from all 15 available single-exposure images covering S82-20

\footnotetext{
3 https://irsa.ipac.caltech.edu/applications/ICORE/
} 
Table 2. Photometric measurements

\begin{tabular}{lc} 
Filter & $m_{A B}$ \\
\hline SDSS u & $>23.89$ \\
SDSS g & $22.17 \pm 0.05(22.9 \pm 0.1)^{a}$ \\
SDSS r & $22.87 \pm 0.10(23.4 \pm 0.2)^{a}$ \\
SDSS i & $23.68 \pm 0.37(23.9 \pm 0.4)^{a}$ \\
SDSS z & $>22.33$ \\
IRAC 3.6 & $21.83 \pm 0.26$ \\
IRAC 4.5 & $22.10 \pm 0.18$ \\
WISE W3 & $>17.1$ \\
WISE W4 & $>14.8$ \\
\hline
\end{tabular}

${ }^{\mathrm{a}}$ The values in parenthesis are corrected for the contribution of emission lines.

(Masci \& Fowler 2009). S82-20 is not detected in the WISE images and in Figure 4 we show the $3 \sigma$ upper limits. The upper limits were computed within the circular aperture of $8 .{ }^{\prime \prime} 25$ and $166^{\prime \prime} 5$ radius, at 12 and $22 \mu \mathrm{m}$, respectively, to account for the wavelength dependent instrumental PSF.

We inspected the HeLMS data at the position of S82-20 and find that the object is not detected in any of the maps, implying $3 \sigma$ upper limits to the flux of $15.6,12.9$, and $10.5 \mathrm{mJy}$ at 250,350 , and $500 \mu \mathrm{m}$, respectively. Finally, the object is not detected in the Faint Images of the Radio Sky (FIRST) project that observed the area at a frequency of $1.4 \mathrm{GHz}$ using the NRAO Very Large Array (VLA). The non detection corresponds to an upper limit on the luminosity of $2.8 \times 10^{29} \mathrm{~W} / \mathrm{Hz}$.

\section{MODELING OF LINE RATIOS}

The LBT spectrum of S82-20 allows us to compute a number of line ratios using rest-frame UV emission lines. These line ratios can be compared with photoionization and shock models to identify the main excitation source responsible for the large Ly $\alpha$ luminosity. In this section we briefly describe the details of the models used to interpret the line ratios in Section 5.

\subsection{AGN NLR model}

We use the most up-to-date grid of the AGN NLR photoionization models of Feltre et al. (2016), presented in Mignoli et al. (2019) . For the star-forming galaxies, we used the models of Gutkin et al. (2016). Both models use the CLOUDY photoionization code Ferland et al. (2013).

The AGN models assume that the gas has an open geometry, appropriate when the covering factor of the gas is small. The AGN ionizing spectrum is described with a series of broken power laws:

$S_{v}= \begin{cases}v^{\alpha} & 0.001 \leq \lambda / \mu m \leq 0.25, \\ v^{-0.5} & 0.25 \leq \lambda / \mu m \leq 10.0, \\ v^{2} & \lambda / \mu m>10.0,\end{cases}$

where $S_{v}$ is the luminosity per unit frequency of the accretion disc. We assume $\alpha=-1.7$ consistent with the value derived by Lusso et al. (2015) in a stacked UV spectrum of 53 luminous quasars at $\mathrm{z} \sim$ 2.4. We keep the AGN accretion-disk bolometric luminosity fixed at $L_{A G N}=10^{45} \mathrm{erg} \mathrm{s}^{-1}$. The model assumes that the NLR has an inner radius of $r_{i n}=90 \mathrm{pc}$, and the internal microturbulence has a characteristic velocity $v_{\text {micr }}=100 \mathrm{~km} \mathrm{~s}^{-1}$. We assume a dust-toheavy element mass ratio, $\xi_{d}$ of 0.3 .

\subsection{SFG model}

For the star-forming galaxies, we used the models of Gutkin et al. (2016), computed following the approach outlined by Charlot \& Longhetti (2001) and using an updated version of the Bruzual \& Charlot (2003) stellar population synthesis model (described in section 2.1 of Gutkin et al. (2016)). A closed spherical geometry is used, which is more appropriate to describe the physical conditions of ionization bounded $\mathrm{H}_{\text {II }}$ regions. We consider the models computed for a standard Chabrier (2003) initial mass function truncated at 0.1 and $100 \mathrm{M}_{\odot}$, a $\mathrm{C} / \mathrm{O}$ abundance ratio $=1(\mathrm{C} / \mathrm{O})_{\odot}(\approx 0.44)$, constant star formation rate and age of $100 \mathrm{Myr}$. We assume the same dust-to-heavy element mass ratio as in the AGN model, $\xi_{d}=0.3$.

In Figure 7 we show different tracks for both AGN and SF-galaxies, corresponding to different values of the ionization parameter at the edge of the Strömgren sphere $\left(U_{S}\right)$, the hydrogen gas density, and the interstellar (gas + dust) metallicity $(Z)$. The parameters varied in the tracks are summarized in table 3 .

\subsection{Shock model}

We also consider the nebular emission produced from radiative shocks computed by Alarie \& Morisset (2019) using the latest shock and photoionization code MAPPING V (Sutherland \& Dopita 2017). The main adjustable parameters in the shock models are the shock velocity (that ranges from 100 to $1000 \mathrm{~km} \mathrm{~s}^{-1}$ ), the metallicity of the gas with the same sets of element abundances as adopted in the SFG and AGN models (Gutkin et al. 2016) described above, the pre-shock gas density (from 1 to $10^{4} \mathrm{~cm}^{-3}$ ), and the transverse magnetic field (from $10^{-4}$ to $10 \mu \mathrm{G}$ ). We chose the same $\mathrm{C} / \mathrm{O}$ ratio adopted for the AGN and star-forming galaxies photoionization models $\left.(\mathrm{C} / \mathrm{O})_{\odot}=0.44\right)$.

The pre-shock density and the transverse magnetic field have less impact on the UV line ratios we study here, compared to the shock velocity and metallicity. Therefore, for simplicity, we fix the preshock density to $1 \mathrm{~cm}^{-3}$, and the transverse magnetic field to 0.5 $\mu G$. In Figure 7 we show the line ratios from both the precursor and shocked gas.

\section{RESULTS}

The optical spectra presented in Figure 1 to 3 confirm that S82-20 is a high-redshift object $(z \sim 3.1)$, identified in our survey because of the excess flux in the $g$-band caused by a strong Ly $\alpha$ emission line. In what follows, we present the measurements performed on the LBT/MODS spectrum (shown in Figure 3), as it is the deepest of the three and covers the longest wavelength range (rest-frame 1000-1950 ̊), unless we say otherwise.

The integrated flux of the $\operatorname{Ly} \alpha$ emission line corresponds to a luminosity of $(3.61 \pm 0.02) \times 10^{44} \mathrm{erg} \mathrm{s}^{-1}$, placing S82-20 among the most luminous Ly $\alpha$ emitters known (Marques-Chaves et al. 2019). These luminosities are commonly associated with AGN activity (see, e.g., Matthee et al. 2017; Sobral et al. 2018; Calhau et al. 2020) and/or powerful Ly $\alpha$ nebulae (e.g., Steidel et al. 2000; Scarlata et al. 2009; Herenz et al. 2020), although exceptionally Ly $\alpha$-bright star-forming galaxies have recently been found (see, e.g., Marques-Chaves et al. 2020). 

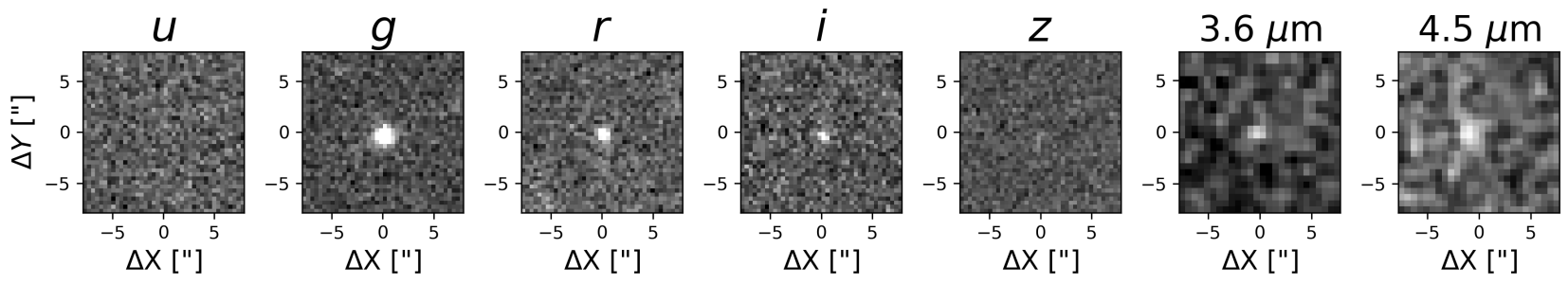

$10^{\circ}$

Observed wavelength $[\mu \mathrm{m}$ ]

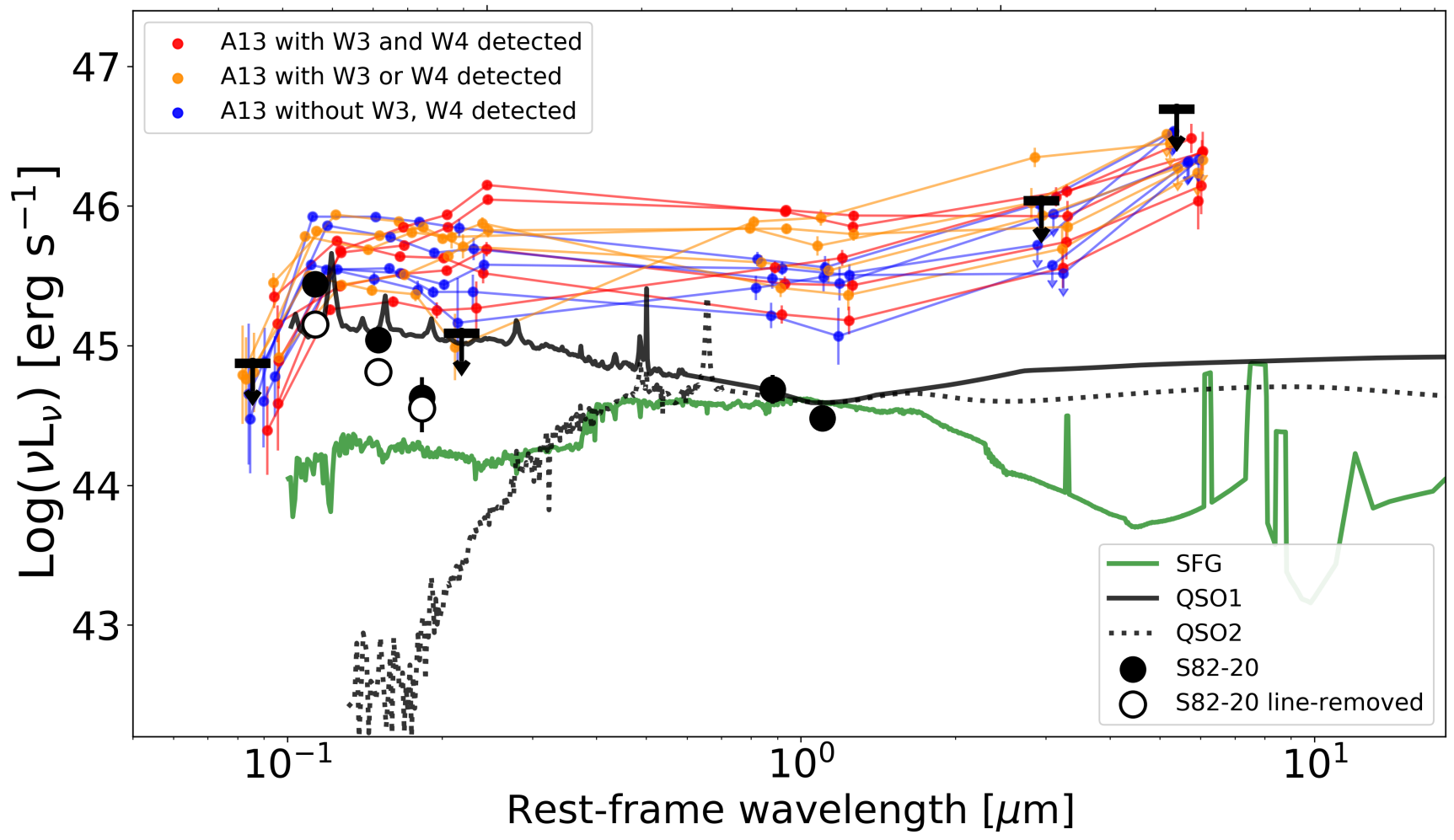

Figure 4. The top panel shows the images of S82-20 in SDSS u, g, r, i, z band and IRAC $3.6 \mu \mathrm{m}, 4.5 \mu \mathrm{m}$. The bottom panel shows the spectral energy distribution (SED) of S82-20 (large points, and upper limits) compared with spectral templates of QSOs (both Type I and Type II) and a star-forming galaxy by Polletta et al. (2007). We also show for comparison the observed SEDs of the A13 Type II QSO candidates, distinguishing between those with both (red points), either one (orange points), and without (blue points) detection at wavelength longer than $6 \mu \mathrm{m}$.

Table 3. Adjustable parameters of the photoionization models.

\begin{tabular}{ccc}
\hline \hline Parameter & AGN & SFG \\
\hline Ionizing spectrum & $\alpha=-1.7$ & $\mathrm{~m}_{u p}=100 \mathrm{M}_{\odot}$ \\
$\log \left(U_{s}\right)$ & $-1.0,-2.0,-3.0,-4.0,-5.0$ & $-1.0,-2.0,-3.0,-4.0$ \\
$\log \left(\mathrm{n}_{H} / \mathrm{cm}^{-3}\right)$ & $2,3,4$ & 2,3 \\
$\mathrm{Z}$ & $0.0001,0.001,0.008,0.017,0.03$ & $0.0001,0.001,0.008,0.017,0.03$ \\
$\xi_{d}$ & 0.3 & 0.3 \\
\hline
\end{tabular}

It is unlikely that the large Ly $\alpha$ luminosity is produced in an extended Ly $\alpha$-nebula. The broad $g$-band image containing the Ly $\alpha$ line reveals that the morphology of S82-20 is compact (see Figure 4) and spatially unresolved in the seeing limited images. This morphology rules out the $\operatorname{Ly} \alpha$ halo interpretation, as these objects have $\operatorname{Ly} \alpha$ sizes of $\approx 100 \mathrm{kpc}$, and are clearly resolved in ground based imaging (e.g., Prescott et al. 2012).

In the rest-frame UV spectrum shown in Figure 3 we identify multiple of high-ionization emission lines. Redward of $\operatorname{Ly} \alpha$, the spectrum shows weak $\mathrm{N} v$ and $\mathrm{O}_{\mathrm{III}}$ ] lines as well as relatively strong 
$\mathrm{C}$ IV, $\mathrm{He}_{\mathrm{II}}$, and $\mathrm{C}$ III] lines. Blueward of $\mathrm{Ly} \alpha$, we detect both emission lines of the $\mathrm{O}$ VI doublets. The presence of these highly ionized ions (particularly the detection of the $\mathrm{O}$ VI) suggest the presence of a hard ionizing spectrum, possibly associated with an AGN. Contribution to the ionization, however, from young stars can not be ruled out, as metal poor dwarf starburst galaxies have been known to show high ionization emission lines of $\mathrm{C}_{\text {IV }}$ and $\mathrm{He}_{\mathrm{II}}$ (Stark et al. 2015; Mainali et al. 2017; Berg et al. 2019; Senchyna et al. 2019, e.g.,).

All lines with sufficient signal-to-noise ratio have consistent line widths, with FWHMs of $\approx 750 \mathrm{~km} \mathrm{~s}^{-1}$. The FWHM of C IV is somewhat larger than that of the other lines, at $838 \pm 10.1 \mathrm{~km} \mathrm{~s}^{-1}$. The doublet, however, is unresolved at the resolution of the LBT spectrum, so we do not consider this larger FWHM significant. Corrected for instrumental resolution the measured FWHM of $750 \mathrm{~km} \mathrm{~s}^{-1}$ corresponds to a velocity dispersion $\sigma=280 \mathrm{~km} \mathrm{~s}^{-1}$. This value is large, but not impossible in galaxies, particularly at $z>2$ (Lehnert, M. D. et al. 2013; Schreiber et al. 2009).

The LBT spectrum shows a faint, but detected, continuum redward of the $\operatorname{Ly} \alpha$ emission line. The flux density of $1.35 \times 10^{-18} \mathrm{erg} \mathrm{s}^{-1} \mathrm{~cm}^{-2} \AA^{-1}$ corresponds to a continuum luminosity $\log \left(L_{1300}\right)=41.7 \pm 0.1 \mathrm{erg} \mathrm{s}^{-1} \AA^{-1}$, or $M_{U V}=-22.7$, and $10^{11.2} L_{\odot}$. The $\mathrm{S} / \mathrm{N}$, however, is too low to search for the presence of absorption lines, that could help pin down the origin of the detected continuum. Combined with the large Ly $\alpha$ luminosity, this continuum implies a rest-frame EW of $754.3 \pm 165.2 \AA$ (see Table 1 ). This value is within $2.5 \sigma$ from the $\mathrm{EW}$ computed using the emission-linecorrected broad band flux $(280.3 \pm 22.2 \AA)$.

In Figure 5 we compare the $\operatorname{Ly} \alpha$ and continuum luminosity of S82-20 with a sample of luminous $\operatorname{Ly} \alpha$ sources. We include both AGN and the only star-forming galaxy known with similar luminosity as our object. For the AGN, we consider the A13 sample of Type II QSOs. This sample was identified by Alexandroff et al. (2013) based on the presence of strong and narrow (with FWHM $<2000 \mathrm{~km} \mathrm{~s}^{-1}$ ) UV emission lines (their "Class A" sample). We also show BOSS-EUVLG1, the Ly $\alpha$ brightest known star-forming galaxy known (Marques-Chaves et al. 2020). For consistency, we remeasured the emission line fluxes and continua for the A13 sample, using the original SDSS spectra and the same technique discussed in Section 2. We only consider A13 objects at $z>2$.6, for which the OVI emission line is covered by the SDSS spectra. For BOSSEUVLG1 we report the published values, assuming the galaxy has a flat spectrum between 1500 and $1300 \AA$ (consistent with the published spectrum, see Figure 1 in Marques-Chaves et al. 2020). The A13 Type II QSO candidates show a general positive correlation between the Ly $\alpha$ luminosity and the rest-frame UV continuum. S8220 is in the upper envelope of this trend, with the UV continuum $\approx 0.7$ dex fainter than what would be predicted based on its Ly $\alpha$ luminosity. Similarly, BOSS-EUVLG1 is an outlier in this correlation, at a much lower Ly $\alpha$ luminosity, given its very bright $\left(\approx 30 L_{U V}^{*}\right) \mathrm{UV}$ continuum.

Due to the resonant nature of the transition, Ly $\alpha$ can be enhanced/suppressed with respect to the continuum by ad hoc geometries of the neutral gas and the presence of even a small amount of dust (Dijkstra et al. 2006). Therefore, in Figure 6, we compare the EW of other lines detected in S82-20 with those of the A13 sample and BOSS-EUVLG1. Clearly, the EW enhancement of S82-20 is present in all UV lines. The Ly $\alpha, \mathrm{C}_{\text {IV }}$ and $\mathrm{C}_{\text {III }}$ EWs of S82-20 are larger than $99 \%, 98 \%$, and $85 \%$ of the obscured QSOs, respectively. The other UV lines identified in BOSS-EUVLG1 behave similarly to the Ly $\alpha$ and are in the lowest quartile of the EW distributions. The bright Ly $\alpha$ luminosity and narrow lines would suggest that S82-20 is powered by an obscured QSO, similar to those in the A13 sample.
The continuum, however, is substantially fainter, implying either a higher obscuration or an enhancement of the emission line fluxes compared to the A13 sample.

In Figure 4 we show the spectral energy distribution of S82-20. Filled and open circles show the direct measurements and those corrected for the emission line contributions, respectively. The restframe UV is remarkably blue. The observed emission-line-corrected color of $g-r=-0.5$ (see Table 2) corresponds to a UV slope $\beta=-3.3_{-0.9}^{+0.6}$, where we followed the convention of parameterizing the continuum as $f_{\lambda} \propto \lambda^{\beta}{ }^{4}$ This continuum is substantially bluer than the typical Type I QSOs, as shown in Figure 4 where we compare the SED of S82-20 to a set of templates from the SWIRE library (Polletta et al. 2007). All templates are normalized to the S82-20's energy at rest-frame $1 \mu \mathrm{m}$. This wavelength was chosen for reference, as it probes a galaxy's stellar mass for objects with either a heavily obscured or low luminosity AGN. Additionally, the effect of dust is minimal at $1 \mu \mathrm{m}$.

In Figure 4 we also show (as the colored points and connecting lines) the SEDs of the A13 Type II QSO candidates at redshifts $2.6<$ $z<3.2$. The WISE data (covering the observed 3.4 to $22 \mu \mathrm{m}$ ) for the A13 sample are taken directly from Alexandroff et al. (2013), while the optical data were retrieved from the SDSS archive. The SDSS photometry for the A13 objects is contaminated by the presence of emission lines so we compare with the filled circles for S8220. At the wavelength of the Ly $\alpha$ emission line ( $g$-band) S82-20 falls square in the middle of the energy range covered by the A13 sample, suggesting that, if indeed powered by an accretion disk, S8220 should have similar ionizing power as these Type II QSOs. As we move to longer wavelengths, however, the energy of S82-20 drops much faster than in the A13 sample. At rest-frame $\approx 1.0 \mu \mathrm{m}, \mathrm{S} 82-20$ is almost an order of magnitude fainter than the bulk of the continuum energies of the A13 sample. This trend continues in the near IR, up to rest-frame $1 \mu \mathrm{m}$, where again the luminosity of S82-20 is one order of magnitude fainter than the bulk of the A13 objects.

\section{DISCUSSION}

The evidence presented in the previous section outline similarities between S82-20 and the population of narrow-line Type II QSOs. S82-20 shares many properties with the sample of Alexandroff et al. (2013), including a similar Ly $\alpha$ luminosity, and width of the emission lines. There are, however, differences that are worth exploring: S8220 has a substantially fainter and bluer continuum than the bulk of Type II QSOs with similar Ly $\alpha$ luminosity, and it is not detected at $5 \mu \mathrm{m}$ rest-frame, where many of the A13 objects are (See Figure 4).

The combination of the fainter continuum with similarly bright emission lines suggest that the S82-20 energy source is an intrinsically powerful QSO (at least as powerful as the A13 objects) but more heavily obscured. In fact, A13 discuss how the obscuration of their objects is not extreme (with $A_{V} \approx 0.5$ ), as revealed by the detection of faint broad wings in rest-frame optical permitted emission lines. The problem with this interpretation, however, is the non-detection of S82-20 in the WISE bands. Indeed, the rest-frame 3-6 $\mu \mathrm{m}$ range probed by WISE should be sensitive to emission from hot dust heated by the energy absorbed in the UV. Energy arguments would suggest that the intrinsic UV luminosity from the accretion disk in the A13 and S82-20 are similar (as they result in the emission of similar

\footnotetext{
4 Written in terms of frequency, the slope corresponds to $\alpha=2.3_{-1.4}^{+1.5}$ (see Equation 2).
} 


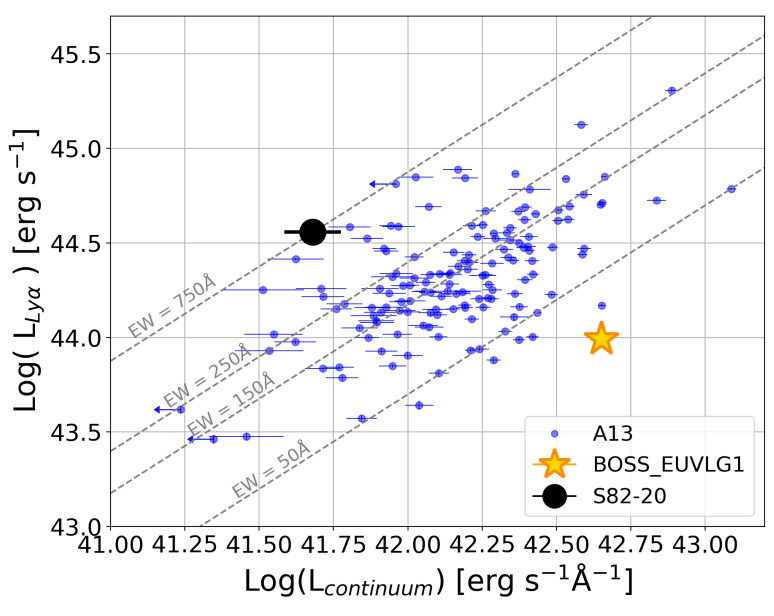

Figure 5. Ly $\alpha$ luminosity versus continuum luminosity at $1300 \AA$, for the A13 sample of Type II QSOs (blue circles), BOSS-EUVLG1 (yellow star) and S82-20 (black circle). The position of S82-20 with respect to the A13 sample indicates that it has a larger continuum obscuration compared to the A13 objects.
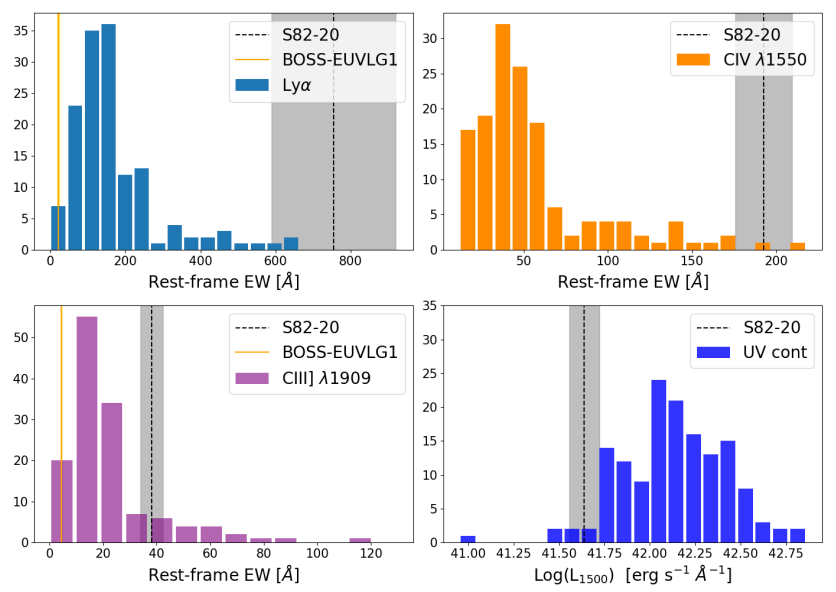

Figure 6. The rest-frame EW of $\left.\operatorname{Ly} \alpha, \mathrm{C}_{\text {IV }}, \mathrm{C}_{\text {III }}\right]$ and the UV continuum luminosity statistics of S82-20 (dash line and gray area), BOSS-EUVLG1 (yellow line) and Type 2 quasar candidates in Alexandroff et al. (2013).

Ly $\alpha$ luminosities). Given the inferred larger obscuration, it is then reasonable to expect that S82-20 should present similar re-emitted luminosities as the A13 in the WISE bands, which is not the case.

Another possibility that could explain the large EWs of the emission lines, is that the lines luminosities are enhanced by another mechanism, possibly star-formation or shocked gas.

\subsection{Type II Emission line ratios}

In Figure 7 we explore the source of ionization of the gas using line ratios computed with the detected rest-frame UV emission lines. Specifically, we show $\mathrm{C}$ IV/He II as a function of N v/C IV, C Iv/C III] and $\mathrm{O}$ vi/C IV, in the left, middle and right panels, respectively. S8220 is marked as a solid black circle, while the A13 Type II QSOs are shown as gray points. Figure 7 shows that the line ratios for
S82-20 are broadly consistent with those of the A13 Type II QSO sample, although S82-20 tends to have a lower $\mathrm{N} \mathrm{v/C} \mathrm{IV} \mathrm{ratio} \mathrm{and}$ a higher $\left.\mathrm{C}_{\mathrm{IV}} / \mathrm{C}_{\mathrm{III}}\right]$ ratio than the bulk of the $\mathrm{A} 13$ emission line selected Type II QSOs. In the left and middle panels, we also show the position of a sample of narrow-line X-ray sources from Nagao, T. et al. (2006b). These objects are located at systematically lower $\mathrm{C}$ Iv/He II ratios compared with the emission line selected Type II QSO sample. Similarly, Type II AGN at redshift $1.5<\mathrm{z}<3$ in Mignoli et al. (2019) objects occupy a similar area of the diagrams.

We compare the observed line ratios with the predictions from photoionization and shocks models described in Section 3. The emission line ratios are not corrected by dust attenuation, and the impact of this is not expected to be significant (Feltre et al. 2016; Hirschmann et al. 2019). Emission line ratios based on $\mathrm{C}_{\text {IV }}$ and $\mathrm{He}_{\text {II }}$ are useful diagnostics to distinguish between nuclear activity and star formation (e.g., Feltre et al. 2016), as the relatively harder AGN ionizing spectrum compared to that of typical star-forming galaxies results in lower $\mathrm{C}$ iv/He II ratios. Figure 7 shows that the Type II AGN models have very distinct $\mathrm{C}_{\text {IV }} / \mathrm{He}_{\text {II }}$ ratios, with $\log \left(\mathrm{C}_{\text {IV }} / \mathrm{He}_{\text {II }}\right)<0.5$. In the model of Mignoli et al. (2019), a smaller inner radius and microturbulence are adopted. These adjustments increase the number of continuum photons that can be absorbed by the gas, enhancing the resonant transitions and therefore increasing the intensity of the high ionization lines such as C IV and N v (See also Kraemer et al. 2007). These models typically reproduce well the line ratios in narrow line AGNs (e.g., Nagao, T. et al. 2006b; Mignoli et al. 2019). Figure 7 shows, however, that the line ratios for S82-20 and the A13 Type II QSO sample are systematically higher than even the most extreme AGN models, but not high enough to be reproduced by models with photoionization by only stars. For both S82-20 and the A13 Type II QSO sample, the $\mathrm{N}_{\mathrm{V}} / \mathrm{C}_{\mathrm{IV}}$ and $\left.\mathrm{C}_{\mathrm{IV}} / \mathrm{C}_{\mathrm{III}}\right]$ ratios, on the other hand, are consistent with photoionization by either AGN or stars. For ionization by stars, the $\mathrm{C}_{\mathrm{IV}} / \mathrm{C}_{\mathrm{III}}$ ] requires a relatively large metallicity of the gas, with $Z \gtrsim 0.017$. For the AGN models, the metallicity constraints are mostly driven by the $\mathrm{N}$ v/C IV ratio, which requires $Z \sim 0.008-0.017$.

The left panel of Figure 7 shows that S82-20 and the A13 Type II QSOs have high values of the $\mathrm{O} v \mathrm{I} / \mathrm{C}_{\mathrm{IV}}$ ratios (larger than 0.2 ), not consistent with photoionization by stars. These high values can only be explained by photoionization from an accretion disk. This is because $\mathrm{O}^{5+}$ ions require photons with energy at least $113.9 \mathrm{eV}$, and the spectra of typical massive stars are not hard enough to produce significant amounts of these high energy photons. We note that line ratios involving the $\mathrm{O}$ vi emission lines have to be regarded as lower limits, as at $z \gtrsim 2$, the $\mathrm{O}$ vi line fluxes can be affected by absorption in the Ly $\alpha$ forest. Correcting for this absorption would move the points to the right, further away from the SF region. As Figure 7 shows, the metallicity in the AGN models is not constrained by this ratio.

The observed high emission line ratios $\left(\log \left(\mathrm{C}_{\text {IV }} / \mathrm{He}_{\mathrm{II}}\right) \geq 0.5\right)$ could be produced in AGN NLRs with extreme conditions: very high ionization parameters and very low metallicity. Such models, however, would still not able to reproduce the $\mathrm{C}$ Iv/He II vs $\mathrm{C}$ iv/ $\mathrm{N} \mathrm{v}$ ratios of both S82-20 and the A13 sample, as shown in Figure 7. The AGN models shown in Figure 7 assume a power law index $\alpha=-1.7$. A harder ionization spectrum $(\alpha=-2.0)$ can produce even higher $\mathrm{C}$ Iv/He II ratio, but still would not the $\mathrm{C}$ Iv/He II vs $\mathrm{C}$ IV/N v ratios. See Mignoli et al. (2019) for the full parameters of the models. In the following sections, we consider various possibilities that could explain the observed properties of S82-20. 

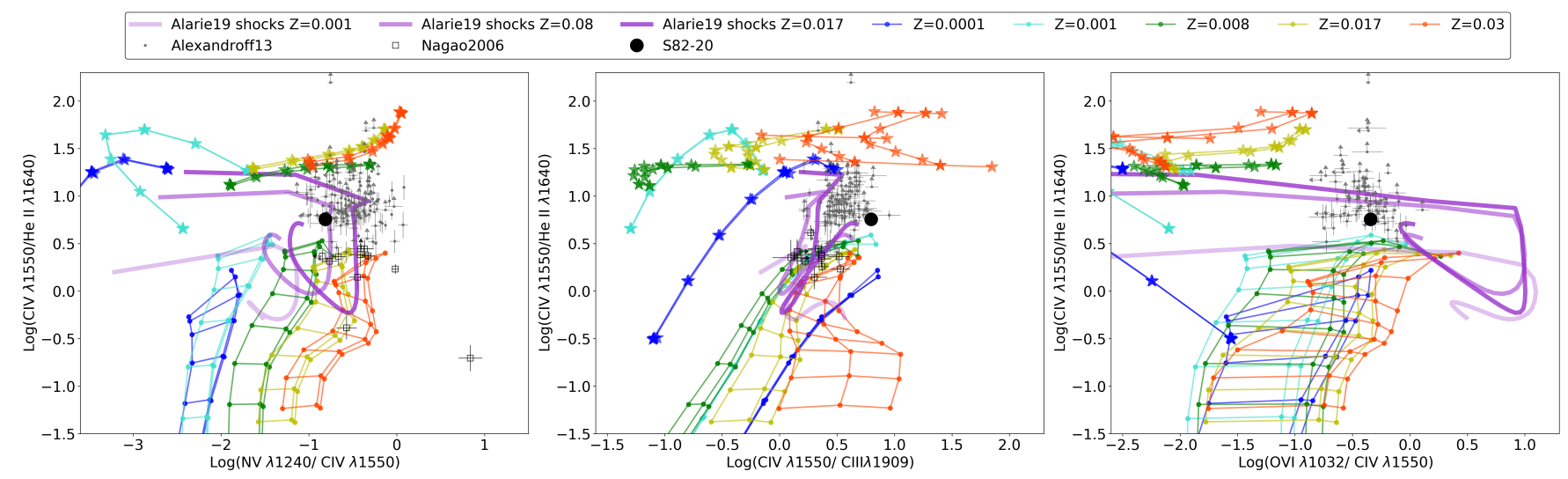

Figure 7. Line ratios diagnostic plots for S82-20 and the sample of Alexandroff et al. (2013). We show $\mathrm{C}_{\mathrm{IV}} / \mathrm{He}_{\mathrm{II}}$ as a function of $\mathrm{N}$ v/ $\mathrm{C}_{\mathrm{IV}} \mathrm{C}$ IV/C $\left.\mathrm{III}\right]$ and $\mathrm{O}$ vi/C Iv, in the left, middle and right panel respectively. We also show line ratios predicted by photoionization models for star-forming galaxies (star markers) from Gutkin et al. (2016) and AGN (dot markers) from Feltre et al. (2016). Finally, the purple lines show the line ratios computed with the shocks model from Alarie \& Morisset (2019).
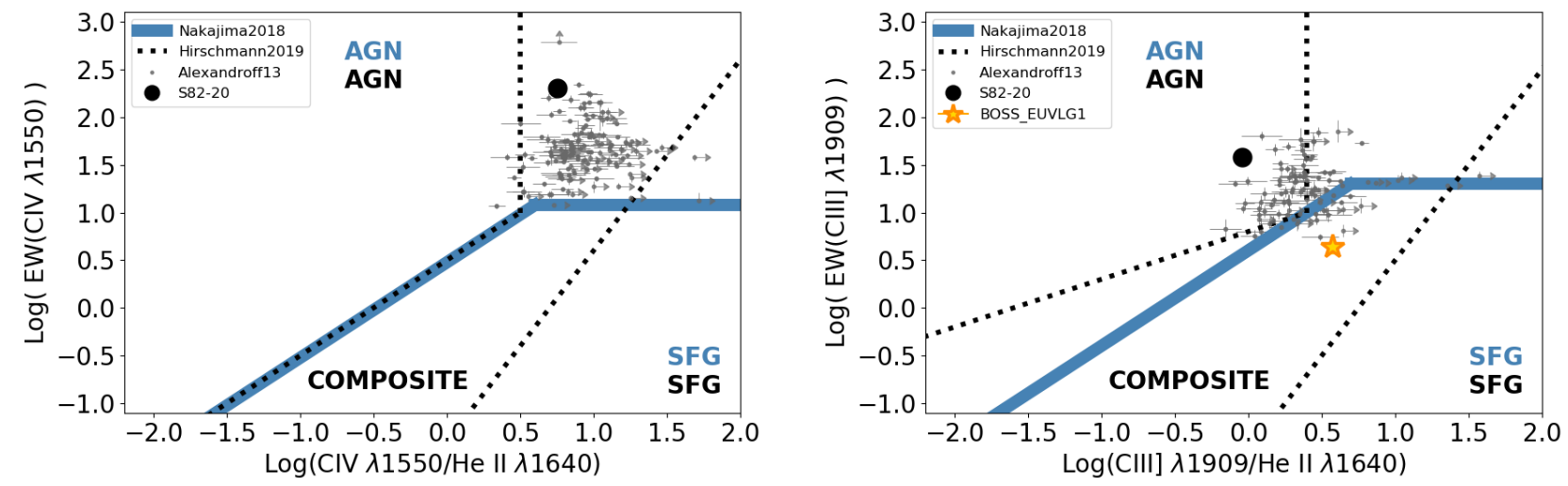

Figure 8. Equivalent width and line ratios diagnostic plots for S82-20 and the sample of Alexandroff et al. (2013). We show the EW(C IV) vs C IV/He II and $\mathrm{EW}\left(\mathrm{C}_{\mathrm{III}}\right]$ ) vs $\mathrm{C}_{\mathrm{III}}$ //He II discriminate lines used in Nakajima, K. et al. (2018) (blue) for star-forming galaxies and AGN, and Hirschmann et al. (2019) (black) for star-forming galaxies, AGN and the composite galaxies.

\subsection{Type I+Type II spectrum}

The high values of the $\mathrm{C}$ IV/He II line ratios observed for the A13 sample and S82-20 are similar to those typically measured in the broad line regions of QSOs (Berk et al. 2001; Alexandroff et al. 2013; Nagao, T. et al. 2006a). Alexandroff et al. (2013) suggest that a possible explanation for these large values is that the measurement of the narrow-component fluxes are contaminated by some partiallyunobscured emission from the broad component. This explanation, however, does not seem to apply to S82-20. In order to increase the $\mathrm{C}$ IV/He II line ratio to the observed values, at least $55 \%$ of the $\mathrm{C}_{\text {IV }}$ emission in the $\pm 900 \mathrm{~km} \mathrm{~s}^{-1}$ range would have to come from a broad component. If this were the case, however, we would have expected to detect the broad component in, e.g., the much brighter Ly $\alpha$ emission line. Additionally, although this explanation could be viable for the A13 objects in which the obscuration is modest (see discussion above), in Figure 5 we show that the continuum of S82-20 is substantially fainter than in the A13 objects, for the given Ly $\alpha$ luminosity, implying that it is substantially more absorbed.

Another possibility is that we observed S82-20 in a temporary, low state. If the accretion rate of SMBH had decreased recently, the UV continuum, the broad emission line component, and the re-emitted infrared will decrease (e.g. MacLeod et al. 2016; Yang et al. 2018). The emission originating from the lower density NLR may have a delay response due to its longer recombination timescale (Denney et al. 2014; LaMassa et al. 2015). Most reported changinglook AGNs do show the Type I spectral features at some point, since the variation of the broad emission component is a decisive element. If such changing of the central engine occurs in a Type II AGN, the accretion disc will be already blocked from the line of sight. Therefore we may not observe variations in the UV and optical spectrum. The first observation (Palomar; October 2013) and the most recent (LBT; October 2020) are about 1.7 years apart in the object's rest frame. The two spectra show similar EWs, line shapes, and line ratios. If the emission lines are emitted from the NLR, the high $\mathrm{C}_{\text {IV }} / \mathrm{He}$ II ratio requires high ionization parameter and microturbulence as described in Section 3. It is unclear, however, whether a lower state AGN would have enough energy to constantly drive this microturbulence. Therefore, with the available data, we can not determine whether S82-20 is a changing look AGN. 


\subsection{Type II+Stars spectrum}

Another possibility is that we are observing the combined emission from gas ionized by both star-formation and AGN. In this scenario, the $\mathrm{O} v \mathrm{a}$ and $\mathrm{Nv}$ emission lines are excited by the AGN spectrum, while both AGN and young stars can produce the C IV emission. In addition to reproducing the unusual line ratios, this explanation would also help to explain the faintness of S82-20 in the IR, as in this case the luminosity of the narrow Ly $\alpha$ emission would not be a good proxy for the intrinsic ionizing power of the accretion disk. In this scenario, the observed blue continuum would be the result of $\mathrm{SF}$, and the AGN-powered line luminosity would be decreased.

The $\mathrm{C}_{\mathrm{IV}}$ and $\left.\mathrm{C}_{\mathrm{III}}\right] \mathrm{EWs}$, in addition to the line ratios, can be used to identify the composite origin of the emission lines, as done by e.g., Nakajima, K. et al. (2018) and Hirschmann et al. (2019). We do this in Figure 8, where we show the classification regions introduced by the two groups. These diagnostics are not conclusive. According to

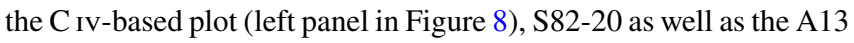
objects, fall in the "composite" part of the diagram. The $\mathrm{C}_{\text {III]-based }}$ diagnostic, however, shows that the A13 objects are located across the separation between AGN and composite spectra, while S82-20 is consistent with AGN photoionization. This discussion highlights how the availability of multiple line ratios often reveals a situation that is more complex than what would appear with limited information. In a composite spectrum scenario, at least $\approx 50 \%$ of the $\mathrm{C}$ IV luminosity would need to be the result of photoionization by stars, in order to move the line ratios from the most extreme AGNs to the observed position .

We can attempt to place a constraint on the stellar mass of the host galaxy, using the two-component SED fitting procedure by Bongiorno et al. (2012), in which the observed optical-to-NIR SED is fitted with a large grid of models created from a combination of AGN and host galaxy templates. In particular, for the AGN component, this procedure adopts the Richards et al. (2006) mean QSO SED, extinguished by applying a SMC-like dust-reddening law (Prevot et al. 1984) of the form: $A_{\lambda} / E(B-V)=1.39 \times \lambda_{\mu \mathrm{m}}^{-1.2}$, while for the galaxy component, a library of synthetic spectra generated using the Bruzual \& Charlot (2003) stellar population synthesis models with declining star formation histories (SFR $\propto e^{-t_{\text {age }} / \tau}$ ) with e-folding times, $\tau$, ranging from 0.1 to $30 \mathrm{Gyr}$ and a grid of ages ranging from $50 \mathrm{Myr}$ to 9 Gyr. Dust extinction of the galaxy component is taken into account using the Calzetti law (Calzetti et al. 2000). For more details, we refer the reader to Bongiorno et al. (2012).The results of the SED fitting code suggest that the host galaxy is a relatively massive, actively starforming system, with $\log \left(M_{*} / M_{\odot}\right)=10.6_{-1.4}^{+0.2}$ and a star-formation rate $\log (S F R)=1.5_{-0.1}^{+0.5}$. The AGN continuum results completely obscured in the rest-frame $U V$, with an estimated $E(B-V)=9$.

An interesting question is where the obscuration of the AGN continuum takes place, as it can happen either close to the disk, in the nuclear torus, or in the host galaxy, following a dusty merger phase (as suggested by, e.g., Hopkins et al. 2008). The available observations disfavor the latter interpretation. First, the UV continuum is very blue, with $\beta=-3.3_{-0.9}^{+0.6}$. Although in principle such blue values of $\beta$ can be produced by young, low-metallicity stars (e.g. Schaerer (2002); Bouwens et al. (2010)), this color would imply that the stellar continuum is not attenuated. Second, the bright Ly $\alpha$ luminosity is difficult to reconcile with a dust-rich environment. If the observed attenuation were due to dust distributed on galaxy-scale, we would expect the Ly $\alpha$ to be substantially suppressed. Consequently, it appears more likely that the obscuration of the AGN continuum and the broad line region happens close to the accretion disk, in a galaxy with an overall low dust content that allows the escape of a substantial amount of $\operatorname{Ly} \alpha$.

\subsection{Shocks}

It is worth exploring an additional mechanism that can result in strong high-ionization emission lines: high velocity radiative shocks. Shocks could result from gas accelerated either by the explosion of massive stars associated with regions of active star-formation (e.g., Yadav et al. 2016; Rosdahl et al. 2016), or in the high-velocity outflows often associated with AGN. In Figure 7 we show the line ratios predicted by the shock models with purple lines. Models with higher metallicity $\left(\sim 0.5-1 \mathrm{Z}_{\odot}\right)$ and a velocity of $200 \mathrm{~km} \mathrm{~s}^{-1}$ are broadly consistent with most of the A13 Type II QSO candidates as well as with S82-20, in all diagnostic plots considered in Figure 7. Therefore even if the mixing source scenarios reproduce the emission line ratio, an unknown fraction of flux can be still contributed from shocks.

Although the shock explanation well reproduces all the emission line ratios, it is not clear whether the entire luminosity of the emission lines can be explained with shocked gas. Additionally, this interpretation still does not shed light on the source of energy that powers the required high velocity gas, as outflows of velocities up to $1000 \mathrm{~s} \mathrm{~km}$ $\mathrm{s}^{-1}$ are observed in both AGN and star-forming galaxies (DiamondStanic et al. 2012; Harrison et al. 2014; Zakamska \& Greene 2014).

\section{CONCLUSIONS}

We presented a luminous $z=3.08$ broad-band selected Ly $\alpha$ emitting object, S82-20, identified through the excess flux in the $g$-band compared to adjacent filters using deep SDSS Stripe 82 data. In this paper we describe the spectroscopic data obtained to confirm the redshift of the source, as well as the spectral energy distribution derived from archival near and mid-infrared data. We use this information to place constraints on the nature of the source ionizing the gas in this object.

The rest-frame UV spectrum shows highly ionized emission lines, including the $\mathrm{C}$ IV $\lambda 1550$ doublet, He II $\lambda 1640$ and $\mathrm{O}$ vi $\lambda 1032$ doublet. The high Ly $\alpha$ luminosity $\left(3.5 \times 10^{44} \mathrm{erg} \mathrm{s}^{-1}\right)$, the absence of broad wings associated with the emission lines, the large emission-line EWs, and the detection of the O vi doublet strongly suggest the interpretation that S82-20 is an obscured Type II QSO.

However, some evidence suggests that the Type II QSO interpretation may not be sufficient to explain all the observables we presented in the paper. The $\mathrm{C}$ IV/He II vs. $\mathrm{N}$ v/C IV ratio is not fully reproduced by AGN photoionization models alone.Additionally, S82-20 is not detected at wavelengths longer than $2 \mu \mathrm{m}$, in tension with the expectation that most of the accretion disk luminosity should be absorbed by the dusty torus and re-emitted isotropically in the far-IR. To fully explain these features, S82-20 may require either some contribution from metal rich star-formation or high velocity shocks. The lack of broad lines could be explained if S82-20 is a QSO in a temporary low state. This scenario, however, may still have problems in reproducing the ratio of the narrow $\mathrm{C}_{\mathrm{IV}} / \mathrm{He}_{\mathrm{II}}$ lines.

Whether the explanation of the observed emission line-ratios in S82-20 is photoionization by a combination of AGN and SF or shock heated gas, this object is a rare example of the short phase of the life of a massive galaxy, in which active SF and accretion onto a supermassive BH coexist (Hopkins et al. 2008). This phase is supposed to be characterized by large obscuration of both the SF episode and the AGN. Our result, however, may have identified a new phase, in which only the nuclear region is heavily obscured, while the galaxy lacks a diffuse dust component. 
We conclude by noting that this object was selected only because the $\operatorname{Ly} \alpha$ emission line made it bright enough to be selected in the $g$-band. Had it been at a different redshift, we may not have selected it. Additionally, this object would have been clearly missed in restframe continuum surveys of QSOs, as Figure 4 shows that it is approximately an order of magnitude fainter than known Type II QSOs at similar redshifts.

\section{ACKNOWLEDGEMENTS}

This research has made use of the NASA/IPAC Infrared Science Archive, which is funded by the National Aeronautics and Space Administration and operated by the California Institute of Technology. Some of the observations reported in this paper were obtained with the Southern African Large Telescope (SALT) under program 20181-MLT-011 (Matthew Hayes). This paper used data obtained with the MODS spectrographs built with funding from NSF grant AST9987045 and the NSF Telescope System Instrumentation Program (TSIP), with additional funds from the Ohio Board of Regents and the Ohio State University Office of Research. M.H. is fellow of the Knut and Alice Wallenberg Foundation. AF acknowledges the support from grant PRIN MIUR2017-20173ML3WW_001. MM and PV acknowledge support from the National Research Foundation of South Africa.

\section{DATA AVAILABILITY}

The data used in this article will be shared on reasonable request to the corresponding author.

\section{REFERENCES}

Alarie A., Morisset C., 2019, Rev. Mex. Astron. Astrofis., 55, 377

Alexandroff R., et al., 2013, Monthly Notices of the Royal Astronomical Society, 435, 3306

Annis J., et al., 2014, The Astrophysical Journal, 794, 120

Antonucci R., 1993, ARA\&A, 31, 473

Asboth V., et al., 2016, MNRAS, 462, 1989

Assef R. J., et al., 2013, ApJ, 772, 26

Berg D. A., Chisholm J., Erb D. K., Pogge R., Henry A., Olivier G. M., 2019, The Astrophysical Journal, 878, L3

Berk D. E. V., et al., 2001, The Astronomical Journal, 122, 549

Bongiorno A., et al., 2012, Monthly Notices of the Royal Astronomical Society, 427,3103

Bouwens R. J., et al., 2010, ApJ, 709, L133

Bruzual G., Charlot S., 2003, MNRAS, 344, 1000

Buckley D. A. H., Swart G. P., Meiring J. G., 2006, in Stepp L. M., ed., Society of Photo-Optical Instrumentation Engineers (SPIE) Conference Series Vol. 6267, Society of Photo-Optical Instrumentation Engineers (SPIE) Conference Series. p. 62670Z, doi:10.1117/12.673750

Burgh E. B., Nordsieck K. H., Kobulnicky H. A., Williams T. B., O’Donoghue D., Smith M. P., Percival J. W., 2003, in Iye M., Moorwood A. F. M., eds, Society of Photo-Optical Instrumentation Engineers (SPIE) Conference Series Vol. 4841, Instrument Design and Performance for Optical/Infrared Ground-based Telescopes. pp 1463-1471, doi: $10.1117 / 12.460312$

Burtscher, L. et al., 2016, A\&A, 586, A28

Calhau J., et al., 2020, Monthly Notices of the Royal Astronomical Society, 493, 3341

Calzetti D., Armus L., Bohlin R. C., Kinney A. L., Koornneef J., StorchiBergmann T., 2000, The Astrophysical Journal, 533, 682

Chabrier G., 2003, PASP, 115, 763
Chambers K. C., et al., 2019, The Pan-STARRS1 Surveys (arXiv: 1612.05560)

Charlot S., Longhetti M., 2001, Monthly Notices of the Royal Astronomical Society, 323, 887

Crawford S. M., et al., 2010, in Observatory Operations: Strategies, Processes, and Systems III. p. 773725, doi:10.1117/12.857000

Croxall K. V., Pogge R. W., 2019, rwpogge/modsIDL: modsIDL Binocular Release, doi:10.5281/zenodo.2561424, https://doi.org/10.5281/ zenodo. 2561424

Denney K. D., et al., 2014, ApJ, 796, 134

Di Matteo T., Springel V., Hernquist L., 2005, Nature, 433, 604

Diamond-Stanic A. M., Moustakas J., Tremonti C. A., Coil A. L., Hickox R. C., Robaina A. R., Rudnick G. H., Sell P. H., 2012, The Astrophysical Journal, 755, L26

Dijkstra M., Haiman Z., Spaans M., 2006, The Astrophysical Journal, 649, 14

Donley J. L., et al., 2012, ApJ, 748, 142

Elitzur M., 2012, The Astrophysical Journal, 747, L33

Elitzur M., Ho L. C., Trump J. R., 2014, Monthly Notices of the Royal Astronomical Society, 438, 3340

Eracleous M., Livio M., Halpern J. P., Storchi-Bergmann T., 1995, ApJ, 438, 610

Fabian A. C., 1999, Monthly Notices of the Royal Astronomical Society, 308, L39

Feltre A., Charlot S., Gutkin J., 2016, Monthly Notices of the Royal Astronomical Society, 456, 3354

Ferland G. J., et al., 2013, Rev. Mex. Astron. Astrofis., 49, 137

Ferrarese L., Merritt D., 2000, The Astrophysical Journal, 539, L9

Gebhardt K., et al., 2000, ApJ, 539, L13

Greene J. E., et al., 2014, The Astrophysical Journal, 788, 91

Gutkin J., Charlot S., Bruzual G., 2016, MNRAS, 462, 1757

Hainline K. N., Hickox R. C., Carroll C. M., Myers A. D., DiPompeo M. A., Trouille L., 2014, The Astrophysical Journal, 795, 124

Hamann F., Shields J. C., Ferland G. J., Korista K. T., 1995, ApJ, 454, 688

Harrison C. M., Alexander D. M., Mullaney J. R., Swinbank A. M., 2014, Monthly Notices of the Royal Astronomical Society, 441, 3306

Hasinger G., 2004, Nuclear Physics B - Proceedings Supplements, 132, 86

Herenz E. C., Hayes M., Scarlata C., 2020, A\&A, 642, A55

Hickox R. C., Alexander D. M., 2018, ARA\&A, 56, 625

Hickox R. C., Myers A. D., Greene J. E., Hainline K. N., Zakamska N. L., DiPompeo M. A., 2017, The Astrophysical Journal, 849, 53

Hirschmann M., Charlot S., Feltre A., Naab T., Somerville R. S., Choi E., 2019, Monthly Notices of the Royal Astronomical Society, 487, 333

Hopkins P. F., Hernquist L., Cox T. J., Di Matteo T., Robertson B., Springel V., 2006, ApJS, 163, 1

Hopkins P. F., Hernquist L., Cox T. J., Kereš D., 2008, The Astrophysical Journal Supplement Series, 175, 356

Jiang L., et al., 2014, The Astrophysical Journal Supplement Series, 213, 12

Keenan F. P., Feibelman W. A., Berrington K. A., 1992, ApJ, 389, 443

Kraemer S. B., Bottorff M. C., Crenshaw D. M., 2007, The Astrophysical Journal, 668, 730

Kramida A., Yu. Ralchenko Reader J., and NIST ASD Team 2019, NIST Atomic Spectra Database (ver. 5.7.1), [Online]. Available: https://physics.nist.gov/asd [2020, August 12]. National Institute of Standards and Technology, Gaithersburg, MD.

LaMassa S. M., et al., 2015, ApJ, 800, 144

Lacy M., et al., 2004, ApJS, 154, 166

Lehnert, M. D. Le Tiran, L. Nesvadba, N. P. H. van Driel, W. Boulanger, F. Di Matteo, P. 2013, A\&A, 555, A72

Li J., et al., 2020, ApJ, 903, 49

Lusso E., Worseck G., Hennawi J. F., Prochaska J. X., Vignali C., Stern J., O'Meara J. M., 2015, MNRAS, 449, 4204

MacLeod C. L., et al., 2016, Monthly Notices of the Royal Astronomical Society, 457, 389

Madau P., Dickinson M., 2014, ARA\&A, 52, 415

Magorrian J., et al., 1998, AJ, 115, 2285

Mainali R., Kollmeier J. A., Stark D. P., Simcoe R. A., Walth G., Newman A. B., Miller D. R., 2017, ApJ, 836, L14 
Marques-Chaves R., et al., 2019, Monthly Notices of the Royal Astronomical Society, 492, 1257

Marques-Chaves R., et al., 2020, Monthly Notices of the Royal Astronomical Society: Letters, 499, L105

Masci F. J., Fowler J. W., 2009, in Bohlender D. A., Durand D., Dowler P., eds, Astronomical Society of the Pacific Conference Series Vol. 411, Astronomical Data Analysis Software and Systems XVIII. p. 67 (arXiv: 0812.4310)

Matthee J., Sobral D., Best P., Smail I., Bian F., Darvish B., Röttgering H., Fan X., 2017, Monthly Notices of the Royal Astronomical Society, 471, 629

Mignoli M., et al., 2019, A\&A, 626, A9

Nagao, T. Marconi, A. Maiolino, R. 2006a, A\&A, 447, 157

Nagao, T. Maiolino, R. Marconi, A. 2006b, A\&A, 447, 863

Nakajima, K. et al., 2018, A\&A, 612, A94

Oke J. B., Gunn J. E., 1982, PASP, 94, 586

Padovani P., et al., 2017, A\&ARv, 25, 2

Penston M. V., Pérez E., 1984, Monthly Notices of the Royal Astronomical Society, 211, 33P

Pogge R., 2019, rwpogge/modsCCDRed 2.0, doi:10.5281/zenodo.2550741, https://doi.org/10.5281/zenodo.2550741

Pogge R. W., et al., 2010, in McLean I. S., Ramsay S. K., Takami H., eds, Society of Photo-Optical Instrumentation Engineers (SPIE) Conference Series Vol. 7735, Ground-based and Airborne Instrumentation for Astronomy III. p. 77350A, doi:10.1117/12.857215

Polletta M., et al., 2007, ApJ, 663, 81

Prescott M. K. M., et al., 2012, The Astrophysical Journal, 752, 86

Prevot M. L., Lequeux J., Maurice E., Prevot L., Rocca-Volmerange B., 1984, A\&A, 132, 389

Raiter Schaerer, D. Fosbury, R. A. E. 2010, A\&A, 523, A64

Richards G. T., et al., 2006, AJ, 131, 2766

Richardson M. L. A., Scannapieco E., Devriendt J., Slyz A., Thacker R. J., Dubois Y., Wurster J., Silk J., 2016, The Astrophysical Journal, 825, 83

Rigby J. R., Rieke G. H., Donley J. L., Alonso-Herrero A., Perez-Gonzalez P. G., 2006, The Astrophysical Journal, 645, 115

Rosdahl J., Schaye J., Dubois Y., Kimm T., Teyssier R., 2016, Monthly Notices of the Royal Astronomical Society, 466, 11

Scarlata C., et al., 2009, The Astrophysical Journal, 704, L98

Schaerer D., 2002, A\&A, 382, 28

Schaerer, D. 2003, A\&A, 397, 527

Schnorr-Müller A., et al., 2016, Monthly Notices of the Royal Astronomical Society, 462, 3570

Schreiber N. M. F., et al., 2009, The Astrophysical Journal, 706, 1364

Senchyna P., Stark D. P., Chevallard J., Charlot S., Jones T., Vidal-García A., 2019, MNRAS, 488, 3492

Silverman J. D., et al., 2008, The Astrophysical Journal, 679, 118

Sobral D., et al., 2018, MNRAS, 477, 2817

Stark D. P., et al., 2015, Monthly Notices of the Royal Astronomical Society, 454, 1393

Steidel C. C., Adelberger K. L., Shapley A. E., Pettini M., Dickinson M., Giavalisco M., 2000, ApJ, 532, 170

Stern D., et al., 2012, The Astrophysical Journal, 753, 30

Sutherland R. S., Dopita M. A., 2017, ApJS, 229, 34

Timlin J. D., et al., 2016, ApJS, 225, 1

Tody D., 1986, in Crawford D. L., ed., Society of Photo-Optical Instrumentation Engineers (SPIE) Conference Series Vol. 627, Instrumentation in astronomy VI. p. 733, doi:10.1117/12.968154

Ueda Y., Akiyama M., Ohta K., Miyaji T., 2003, ] 10.1086/378940, 598, 886

Urry C. M., Padovani P., 1995, PASP, 107, 803

Veilleux S., Osterbrock D. E., 1987, ApJS, 63, 295

Vito F., et al., 2017, Monthly Notices of the Royal Astronomical Society, 473, 2378

Wright E. L., et al., 2010, AJ, 140, 1868

Yadav N., Mukherjee D., Sharma P., Nath B. B., 2016, Monthly Notices of the Royal Astronomical Society, 465, 1720

Yang Q., et al., 2018, ApJ, 862, 109

Zakamska N. L., Greene J. E., 2014, Monthly Notices of the Royal Astronomical Society, 442, 784
This paper has been typeset from a $\mathrm{T}_{\mathrm{E}} \mathrm{X} / \mathrm{LAT} \mathrm{E} \mathrm{X}$ file prepared by the author. 